\title{
A photothermal Mach-Zehnder interferometer for measuring caffeine and proteins in aqueous solutions using external cavity quantum cascade lasers
}

\author{
Christian Kristament, ${ }^{a}$ Andreas Schwaighofer, ${ }^{\mathrm{a}}$ Milagros Montemurro, ${ }^{\mathrm{a}, \mathrm{b}}$ Bernhard Lendl, ${ }^{* a}$ \\ anstitute of Chemical Technologies and Analytics, Technische Universität Wien, Getreidemarkt \\ 9/164, A 1060 Vienna, Austria \\ baboratorio de Desarrollo Analítico y Quimiometría, FBCB, \\ Universidad Nacional del Litoral-CONICET, Ciudad Universitaria, \\ 3000 Santa Fe, Argentina
}

\begin{abstract}
Copyright 2018 Society of Photo-Optical Instrumentation Engineers (SPIE). One print or electronic copy may be made for personal use only. Systematic reproduction and distribution, duplication of any material in this publication for a fee or for commercial purposes, and modification of the contents of the publication are prohibited.
\end{abstract}

The published version of this accepted manuscript is available under: C. Kristament, A. Schwaighofer, M. Montemurro, B. Lendl, "A photothermal Mach-Zehnder interferometer for measuring caffeine and proteins in aqueous solutions using external cavity quantum cascade lasers," Proc. SPIE 10490, Biomedical Vibrational Spectroscopy 2018: Advances in Research and Industry 104900M (March 13, 2018). DOI: 10.1117/12.2290603.

\begin{abstract}
One of the advantages of mid-IR spectroscopy in biomedical research lies in its capability to provide direct information on the secondary structure of proteins in their natural, often aqueous, environment. One impediment of direct absorption measurements in the correspondent spectral region is the strong absorbance of the native solvent $\left(\mathrm{H}_{2} \mathrm{O}\right)$. In this regard, the advent of broadly-tunable external cavity quantum cascade lasers (EC-QCL) allowed to significantly increasing the optical path length employed in transmission measurements due to their high spectral power densities. Low measured $\mathrm{S} / \mathrm{N}$ ratios were improved by elaborated data analysis protocols that corrected mechanical flaws in the tuning mechanism of ECQCLs and allow for S/N ratios comparable to research grade FTIR spectrometers.

Recent development of new optical set-ups outpacing direct absorption measurements led to further advancements. We present a dedicated Mach-Zehnder interferometer for photothermal measurements in balanced detection mode. In this highly sensitive design, the interferometer is illuminated by a HeNe laser to detect the refractive index change induced by the heat insertion of the EC-QCL. Here, we present photothermal phase shift interferometry measurements of caffeine in ethanol as well as casein in water. Further, the dependency of the signal amplitude on varying modulation frequencies was investigated for different liquids.
\end{abstract}

Keywords: quantum cascade laser, photothermal spectroscopy, Mach-Zehnder interferometer, mid-infrared, balanced detection, proteins

\section{INTRODUCTION}

Spectroscopy in the mid-IR region offers rich and specific chemical information in a non-destructive and label-free manner. The emerging availability of quantum cascade lasers (QCL) has opened up new possibilities for mid-IR spectroscopy and these new light sources have been extensively applied in different fields. ${ }^{1-3}$ QCLs combine high emission power, stable operation at room temperature and versatility in pulsed and CW mode with wide modulation capability. The high spectral power densities and broad spectral tunability of external cavity QCLs (EC-QCL) allowed to significantly increase the path lengths for laser-based IR transmission measurements of liquid-phase samples compared to conventional FTIR 
spectroscopy. ${ }^{4-6}$ Furthermore, the commercial availability of QCLs revitalized the implementation of spectroscopic techniques that measure absorption in an indirect way. In classical direct absorption spectroscopy, the light is attenuated by the sample and the transmitted light is detected. Consequently, experimental implementation and optical detection can be challenging, particularly for highly absorbing samples. In direct absorption spectroscopy, the signal of interest (absorbance) scales with the logarithm of the emission intensity of the light source. By contrast, application of indirect detection schemes truly harnesses the high spectral power densities provided by QCLs, because the detected signal generated by the sample's absorption is directly proportional to the laser emission intensity. Indirect absorbance detection schemes include photoacoustic and photothermal (PT) spectroscopy. ${ }^{7,8}$ In these approaches, the modulated IR pump laser intensity is partly or entirely absorbed by the medium, followed by a temperature change and consequential alteration of its refractive index. Photoacoustic techniques measure the pressure of the thermal waves induced by the periodic temperature change in the sample after absorption. Photothermal techniques aim at detecting changes in the refractive index. This can be achieved by different approaches, e.g. photothermal deflection (mirage-effect), thermal lensing and photothermal phase shift interferometry, which is considered as most sensitive. ${ }^{7}$ In the latter technique, the analyte is placed in one beam of an interferometer (e.g Mach-Zehnder, Fabry-Pérot, Michelson or Jamin). Irradiation of the pump laser beam leads to a variation in the refractive index that causes a change in the wave phase of the probe laser. This technique has been applied for sensitive concentration measurements of gases, ${ }^{9}$ flow rate measurements of liquids and gases as well as surface absorption measurements. ${ }^{10}$

Here, we introduce a sensitive homodyne detection scheme for measuring the refractive index change in liquid-phase samples by a Mach-Zehnder interferometer (MZI), which is illuminated by a HeNe laser as a probe laser. An EC-QCL is employed as pump laser. To the best of our knowledge, this is the first report of a QCL employed in a photothermal interferometry setup for analysis of liquid samples. Previously, QCLs have been used to measure photothermal deflection of a surface-deposited monolayer, ${ }^{8}$ molecular recognition ${ }^{11}$ and standoff detection. ${ }^{12,13}$ Employing the presented approach, photothermal spectra are acquired while exploiting the high emission power provided by QCLs for sample excitation, whereas employing a HeNe probe laser as well as inexpensive optics and sensitive detectors in the visible spectrum range in contrast to expensive components required for the mid-IR region. In principle, this technique can be adapted for detection of different analytes with appropriate matching of the QCL pump laser to the corresponding molecular absorption frequencies. In this work, we present photothermal phase shift interferometry measurements of caffeine in ethanol as well as casein in water. Furthermore, the dependency of the signal amplitude on varying modulation frequencies was investigated for different liquids.

\section{THEORETICAL BACKGROUND}

\subsection{Principals of photothermal spectroscopy}

Photothermal spectroscopy is based on the absorption of light and subsequent refractive index changes. The absorbed light is transformed into heat, which causes an inhomogeneous distribution of the temperature close to the irradiation point. The induced temperature variation also leads to transient changes in the refractive index of the medium and consequently the optical path length. This anisotropic effect leads to formation of an optical element similar to lenses, which in general consists of a material with a refractive index different from the surrounding media. In the case of a laser-based setup, a pump laser is used to heat the sample and a probe laser is employed to measure the refractive index change..$^{10,14,15}$

Generally, the processes involved in photothermal spectroscopy can be separated into three stages:

Stage 1: Absorption of pump laser light.

Stage 2: Dissipation of the absorbed energy as heat into the medium and ensuing change in the refractive index.

Stage 3: Impact of the thermooptical element on transmitted light of the probe laser.

As the calculation of stages 1 and 3 are straightforward in comparison to the temperature distribution (stage 2), the mathematical key problem is to obtain a solution for the changes in the temperature distribution induced by the pump laser. The three-dimensional differential equation of thermal diffusion, in the most general case of a flowing medium, gives this spatial heat distribution:

$$
\frac{\partial T(\boldsymbol{r}, t)}{\partial t}=D \nabla^{2} T(\boldsymbol{r}, t)-v_{x} \frac{\partial T(\boldsymbol{r}, t)}{\partial x}+\frac{1}{\rho C_{p}} Q(\boldsymbol{r}, t)
$$


where $\mathrm{D}$ is the diffusivity, $\rho$ is the specific density, $\mathrm{T}$ is the temperature change, $\mathrm{Q}$ is the heat produced by the absorbed laser intensity, $C_{p}$ is the specific heat capacity of the sample and $v_{x}$ is the flow velocity of the sample in one direction. For a modulated temperature change, the signal $\mathrm{Q}$ is given by equation (3) if we assume a Gaussian laser beam, which is shown by $^{14}$

$$
\begin{gathered}
I(\boldsymbol{r})=\frac{2 E_{0}}{\pi a^{2}} \exp \left(-\frac{2 r^{2}}{a^{2}}\right) \\
Q(\boldsymbol{r}, t)=\alpha I(\boldsymbol{r}, t)=\left\{\begin{array}{cc}
\frac{2 \alpha E_{0}}{\pi a^{2} t_{0}} \exp \left(-\frac{2 r^{2}}{a^{2}}\right) & \text { for } 0 \leq t \leq t_{0} \\
0 & \text { for } t>t_{0}
\end{array}\right.
\end{gathered}
$$

Herein, $\alpha$ is the linear absorption coefficient of the sample and $\mathrm{E}_{0}$ denotes the total energy per pulse. Equation (1) without any further assumptions has no analytical solution, so the following assumptions are needed to obtain an estimation for the temperature change in the flow cell: (i) there is no flow within the sample cell, (ii) the entire pump beam power is absorbed and also diffused linearly during one modulation period at the probe laser beam overlap, (iii) the duty cycle of the pump laser is $50 \%$ and (iv) there is uniform power distribution across the beam (cylindrical beam in z-direction). In these cases, the temperature change can be estimated by ${ }^{16}$

$$
\Delta T=\frac{\alpha E_{l}}{4 \pi a^{2} \rho C_{p} f}
$$

Equation (4) shows the temperature dependency on the modulation frequency $\mathrm{f}$, the laser power $\mathrm{E}_{\mathrm{l}}$, the heat capacity, and the density of the sample $\rho$. The temperature change caused by the pump laser leads to a refractive index change which is given by equation (5) and (6). ${ }^{9}$

$$
\begin{gathered}
n(\boldsymbol{r}, t)=n_{0}+\left.\frac{\partial n}{\partial T}\right|_{T_{0}} T(\boldsymbol{r}, t) \\
\Delta n=\left(\frac{n-1}{T_{0}}\right) \Delta T
\end{gathered}
$$

\subsection{Photothermal interferometry}

The induced change of the refractive index leads to a change in the effective optical path, which can be measured as a phase shift of the probe laser by an interferometer. This phase shift change is given by

$$
\Delta \varphi=\frac{2 \pi l \Delta n}{\lambda} .
$$

In this work, a Mach-Zehnder interferometer featuring two detectors (balanced detection) was used employing a HeNeLaser $(633 \mathrm{~nm})$ as probe laser. Equation (8) shows the relationship of the measured signal at the two detectors and the phase shift $\Delta \varphi .^{17}$

$$
\text { Detector } 1: I=4 I_{0} \cos ^{2}\left(\frac{\Delta \varphi}{2}\right) \quad \text { Detector } 2: I=4 I_{0} \sin ^{2}\left(\frac{\Delta \varphi}{2}\right)
$$

In the interferometer, one mirror is mounted on a piezo actuator in order to adjust the two arms to the position where both output signals are equal. At this so-called quadrature point, the phase shift between the two arms is an odd multiple of $\pi / 2$ (see Figure 1A). ${ }^{16}$ 

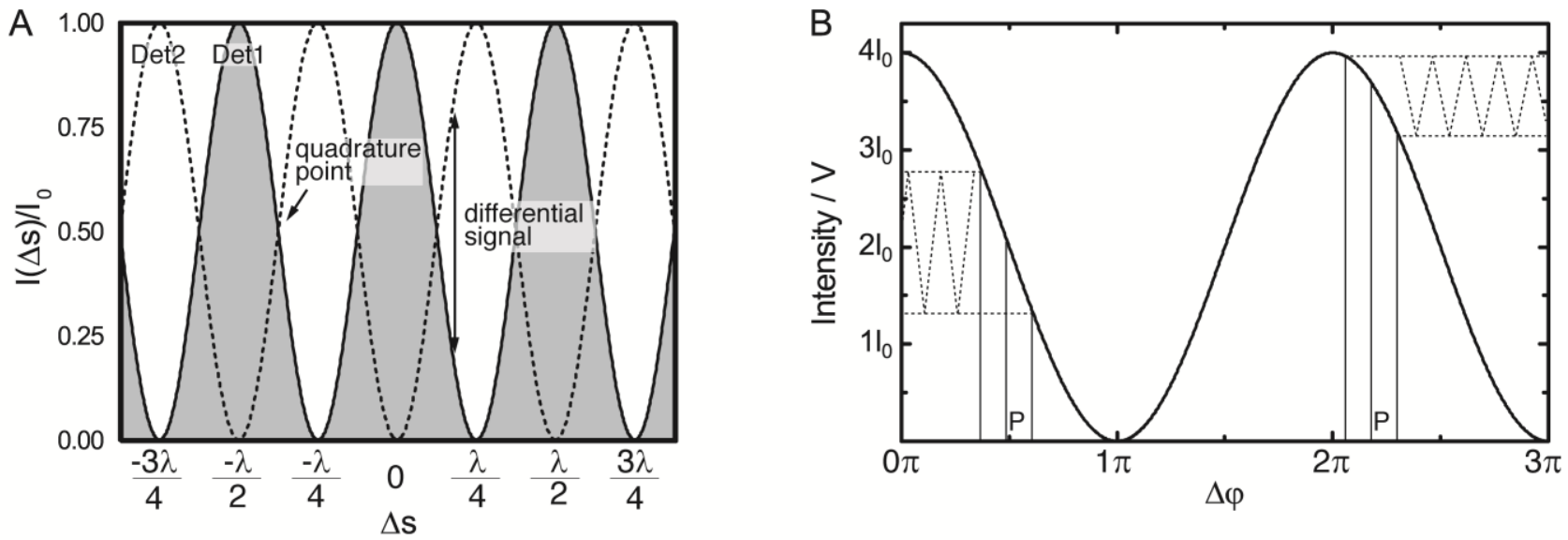

Figure 1: (A) Schematic depicting the intensity response on the two detectors at varying phase shifts, where $\Delta \mathrm{s}$ denotes the path length difference between the two interferometer arms $\left(\Delta \varphi=\frac{4 \pi}{\lambda} \Delta s\right) \cdot{ }^{18}$ At the quadrature point, the interferometer shows its highest sensitivity and stability. (B) Signal size in relation to the operating point $\mathrm{P}$ of the interferometer at a constant phase shift induced by the sample.

Figure 1B shows the signal dependency on the working point of the interferometer. ${ }^{17}$ The output signal $\Delta \mathrm{I}_{0}$ of the system strongly decreases apart from the quadrature point when working at a constant phase shift, because at this operation point the slope is highest and decreases towards the maximum and minimum of the $\sin ^{2}$ proportional output signal of the interferometer. At the quadrature point, the differential signal between the two detectors is zero. ${ }^{18}$ If a modulated pump laser beam irradiates into a sample of one interferometer arm, the differential signal shows a modulated phase shift. This signal can be detected by a lock-in amplifier and is proportional to the absorption coefficient of the sample. The bandwidth of the control loop, which stabilize the quadrature point, must be lower than the pump beam modulation because otherwise the signal of the sample would be cancelled out. ${ }^{17}$ This so-called balanced detection offers the benefit that power fluctuation of the probe laser and common mode noise are highly rejected. ${ }^{9}$ A further advantage is that the phase shift signal is twice as large as with a single detector, because the signal of one detector decreases while the other detectors signal increases by the same amount and the difference is measured.

\section{IMPLEMENTATION OF PT-MACH-ZEHNDER INTERFEROMETRY}

The experimental setup for photothermal interferometric measurements of liquid samples employing an EC-QCL is shown in Figure 2. The Mach-Zehnder interferometer is illuminated by a HeNe laser $(633 \mathrm{~nm})$ and consists of two beam splitters (white rectangles) built for the visible range. One of the two mirrors is a beam combiner (orange), which combines the beam of the pump EC-QC laser beam with the visible beam (probe laser). The other mirror (grey) is mounted on a piezo electric actuator to control the perfect working point of the interferometer (quadrature point), where the interferometer has its highest sensitivity regarding the phase shift that is proportional to the analyte concentration. An EC-QCL from Daylight Solutions (San Diego, USA) is used in CW mode as a pump laser to heat the sample in the spectral region of 1730-1565 $\mathrm{cm}^{-1}$. This laser is modulated with a mechanical chopper, causing a modulated phase shift due to the absorption coefficient of the measured sample. The visible laser is detected by two silicon detectors (DET10A, Thorlabs) at each output of the interferometer in a differential configuration. The differential signal is measured by a self-built lock-in amplifier and a ADS1256 24bit-ADC from Texas Instruments (National Instruments Corp., Austin, USA).

For improved temperature matching between the two liquid cells, they were incorporated in one single aluminum block. To avoid temperature drifts in the cells, a TEC-controller is used to stabilize the temperature of the cells at a temperature of $24{ }^{\circ} \mathrm{C}$. The flow cells were equipped with two $\mathrm{CaF}_{2}$ windows separated by a $25 \mu \mathrm{m}$-thick spacer. To minimize the influence of air flows und water vapor, the setup was housed with black hardboards, and was constantly purged with dry air. Black hardboards are employed to eliminate the influence of ambient visible light on the interferometric measurement. 


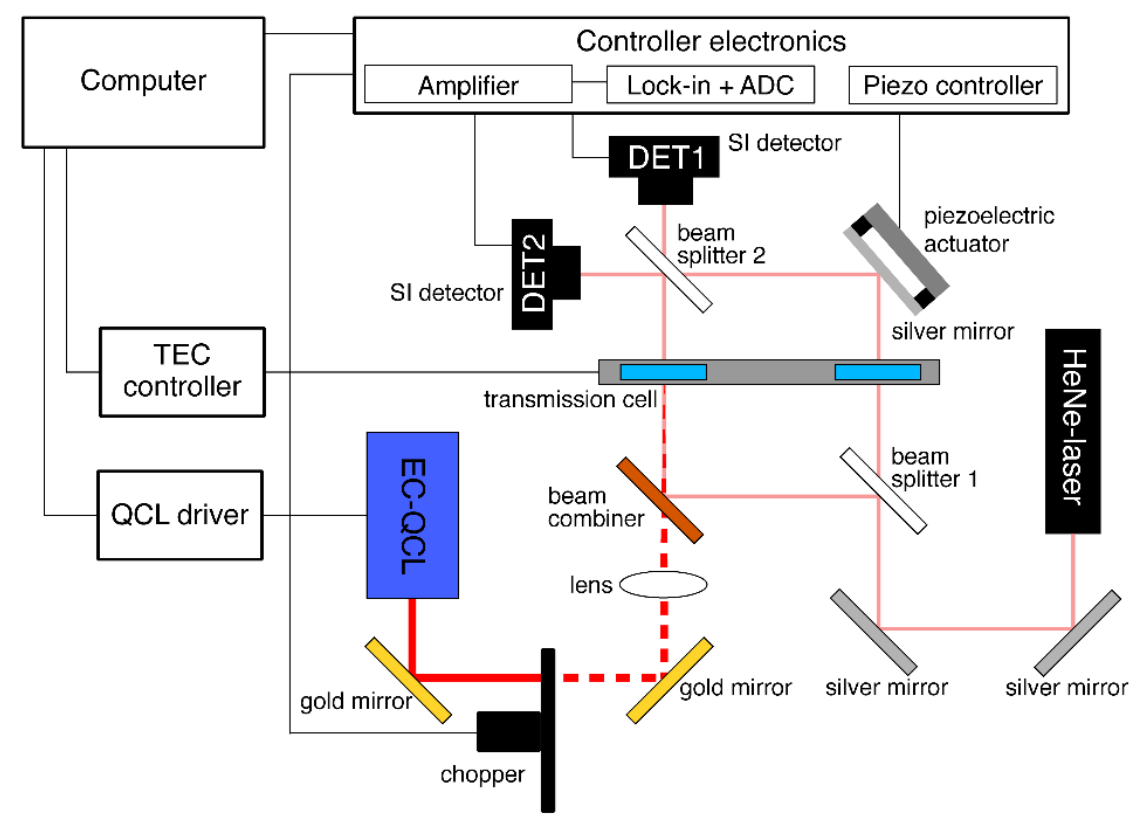

Figure 2: Schematic of the developed PT-MZI setup.

For each scan of the background and sample spectra, 7000 data points were recorded during the EC-QCL tuning time of $3 \mathrm{~s}$. For each spectrum, 20 scans (total acquisition time: $200 \mathrm{sec}$ ) were recorded and averaged. The resulting spectra were filtered by a FFT-filter (cutoff: $10 \mathrm{~Hz}$ ). The liquid samples were handled by an automatic flow injection system, consisting of a 10-way selection valve from VICI (Valco Instruments Co. Inc., Schenkon, Switzerland), a 5 mL Cavro Xcalibur syringe pump from Tecan (Männedorf, Switzerland) and PTFE tubings. During spectra acquisition, the sample was pumped through the sample cells at a flow rate of $0.5 \mathrm{~mL} \mathrm{~min}^{-1}$. Setup control and data acquisition was performed by a custom-made LabView-based GUI.

FTIR reference spectra were recorded using a Bruker 80v FTIR spectrometer (Ettlingen, Germany) equipped with a liquid nitrogen cooled $\mathrm{HgCdTe}$ (Mercury Cadmium Telluride) detector in a transmission cell with two $\mathrm{CaF}_{2}$ windows separated by a $25 \mu \mathrm{m}$-thick spacer. During measurements, the spectrometer was flushed with dry air for at least 10 min prior to spectrum acquisition. Spectra were acquired with a spectral resolution of $4 \mathrm{~cm}^{-1}$ in double-sided acquisition mode. A total of 32 scans were averaged per spectrum, which was calculated using a Blackman-Harris 3-term apodization function and a zero filling factor of 2. Spectra were analysed using the software package OPUS 7.2 (Bruker, Ettlingen, Germany). If necessary, absorption bands of water vapour in the atmosphere were subtracted.

\section{RESULTS AND DISCUSSION}

\subsection{Dependency of the PTI amplitude on the modulation frequency}

PT-MZI amplitudes were recorded of pure ethanol and $1.5 \mathrm{~g} \mathrm{~L}^{-1}$ caffeine in ethanol (for structure see Figure 3A) at modulation frequencies between 30 and $230 \mathrm{~Hz}$ (Figure 4). Since ethanol does not exhibit vibrational bands in the investigated spectral region, the shape of the PTI signal of pure ethanol matches the emission power profile of the 
A<smiles>Cn1c(=O)c2c(ncn2C)n(C)c1=O</smiles>

B<smiles>[R]C(N)C(=O)NC(C)C(=O)NC(C)C([R])N</smiles>

Figure 3: (A) Molecular structure of caffeine. (B) Schematic of the primary structure of proteins with its amino acids held together by peptide bonds. The protein amide I IR-band is mainly associated with the $\mathrm{C}=\mathrm{O}$ stretching vibrations of the peptide bond.
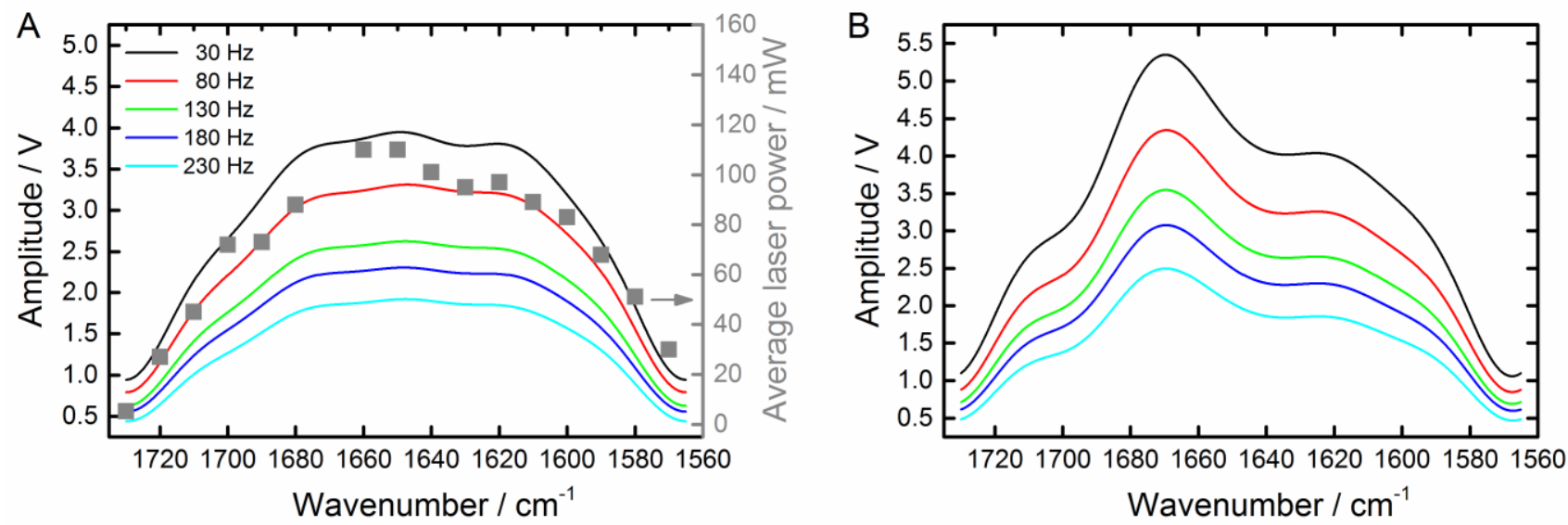

Figure 4: PTI signals of (A) pure ethanol and (B) $1.5 \mathrm{~g} \mathrm{~L}^{-1}$ caffeine in ethanol measured at different modulation frequencies.

EC-QCL (Figure 4A). In presence of caffeine, two distinct peaks are present at approx. 1660 and $1710 \mathrm{~cm}^{-1}$ corresponding to $\mathrm{C}=\mathrm{O}$ absorption bands of caffeine (Figure $4 \mathrm{~B}$ ). ${ }^{19}$ As expected, the PTI amplitude decreases with increasing modulation frequency, because under these experimental conditions the non-optical response cannot take place to its full extent. In addition, the dependence of the PTI amplitude on the modulation frequency was investigated for different media. PT-MZI spectra were recorded of ethanol and water at modulation frequencies between 30 and $230 \mathrm{~Hz}$ and the maximum amplitude was evaluated (Figure 5A). In agreement with equations (4-7), the progression follows an evolution proportional to 1/f at modulation frequencies above a certain threshold frequency. Below this frequency, the amplitude levels off to lower values, because a thermal equilibrium is reached between the irradiated laser energy and the dissipated energy by diffusion in the medium. The threshold frequency as well as the achievable height of the PTI signal depends on the physical properties of the respective medium i.e. the threshold frequency in water is lower compared to ethanol due to its higher heat capacity and density. Unfortunately, in the current configuration of the setup, the band width of the signal detection controller is not capable of performing measurements with modulation frequencies lower than $30 \mathrm{~Hz}$. Furthermore, as depicted in Figure $5 \mathrm{~A}$, the PTI amplitude at a given frequency is higher for ethanol than the one obtained for water. The significantly higher absorption coefficient of water compared to ethanol in the investigated spectral region (water HOH-bending band: $1643 \mathrm{~cm}^{-1}, \alpha=21.8 \mathrm{~L} \mathrm{~mol}^{-1} \mathrm{~cm}^{-1}$ vs ethanol: $\left.\alpha=\sim 0.8 \mathrm{~L} \mathrm{~mol}^{-1} \mathrm{~cm}^{-1}\right)^{20}$ in combination with the higher molar concentration of water $\left(55.56 \mathrm{~mol} \mathrm{~L}^{-1}\right)$ compared to ethanol $\left(17.2 \mathrm{~mol} \mathrm{~L}^{-1}\right)$ leads to absorption of the irradiated laser light within a few micrometers in the liquid film. This results in a significantly lower effective path length for interaction with the probe laser (see schematic in Figure 5B). Further, also the higher heat capacity (4.18 vs. $\left.2.4 \mathrm{~kg} \mathrm{~m}^{2} \mathrm{~K}^{-1} \mathrm{~s}^{-2}\right)$ and higher density $(998 \mathrm{vs}$. $789 \mathrm{~g} \mathrm{~L}^{-1}$ at $20^{\circ} \mathrm{C}$ ) of water lowers the PTI signal in comparison to ethanol. Another reason for the higher PTI signal of water is the significantly higher slope $\frac{d n}{d T}$ for ethanol (see equation (4) and (6) ${ }^{21,22} \mathrm{An}$ in-depth investigation of the influence 
of the density, heat capacity and absorption index as well as experimental conditions such as the flow velocity on the PTI amplitude will be performed in a future study.

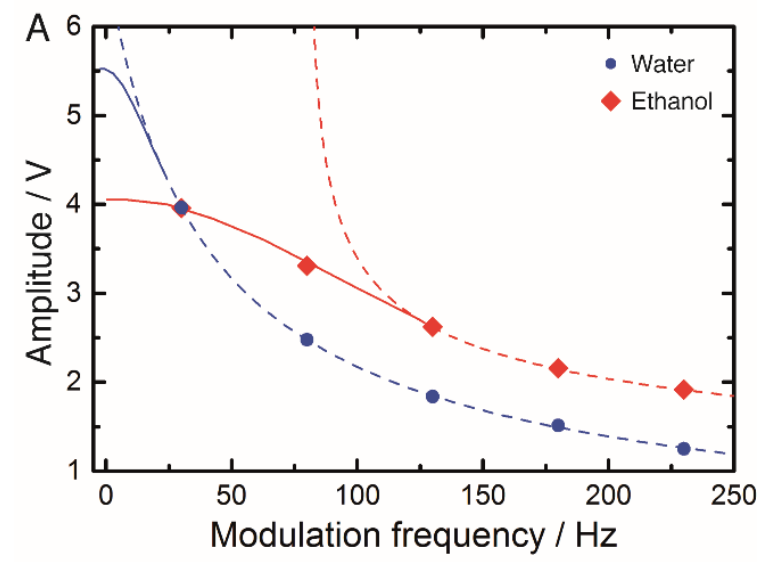

B

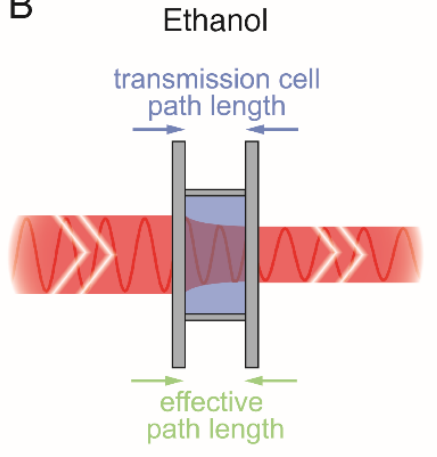

Water

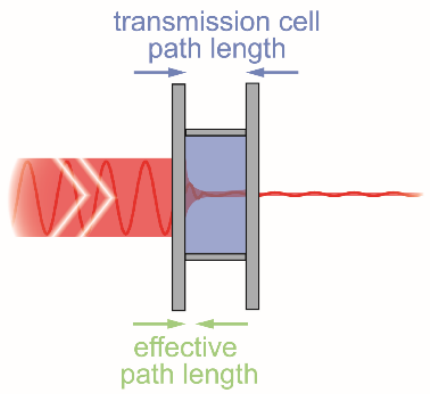

Figure 5: (A) Dependence of the PTI amplitude on the modulation frequency for water and ethanol. Dashed lines show hyperbolic fits with three parameters of the measured data points. Solid lines show levelled progression below threshold frequency. (B) Schematics illustrating the effect of different absorption coefficients of ethanol and water on the effective path length in PT-MZI measurements.

\subsection{PT-MZI spectra of caffeine in ethanol}

Normalized PT-MZI spectra of caffeine in ethanol were obtained by division of the PTI amplitudes of the ethanolic caffeine solutions with the measured data for pure ethanol. After normalization, the PTI spectra obtained at different modulation frequencies show similar amplitudes independent of the modulation frequency used for data acquisition (Figure 6A). The normalized PTI spectra show clear bands at approx. 1660 and $1710 \mathrm{~cm}^{-1}$, characteristic for $\mathrm{C}=\mathrm{O}$ absorption bands of caffeine. ${ }^{19}$ For comparison, a FTIR spectrum of caffeine in ethanol is shown in Figure $6 \mathrm{~B}$.

Moreover, PTI amplitudes were recorded at different caffeine concentrations at a modulation frequency of $230 \mathrm{~Hz}$ (Figure 7A). The amplitude values show linear progression with increasing caffeine concentrations (Figure 7B).
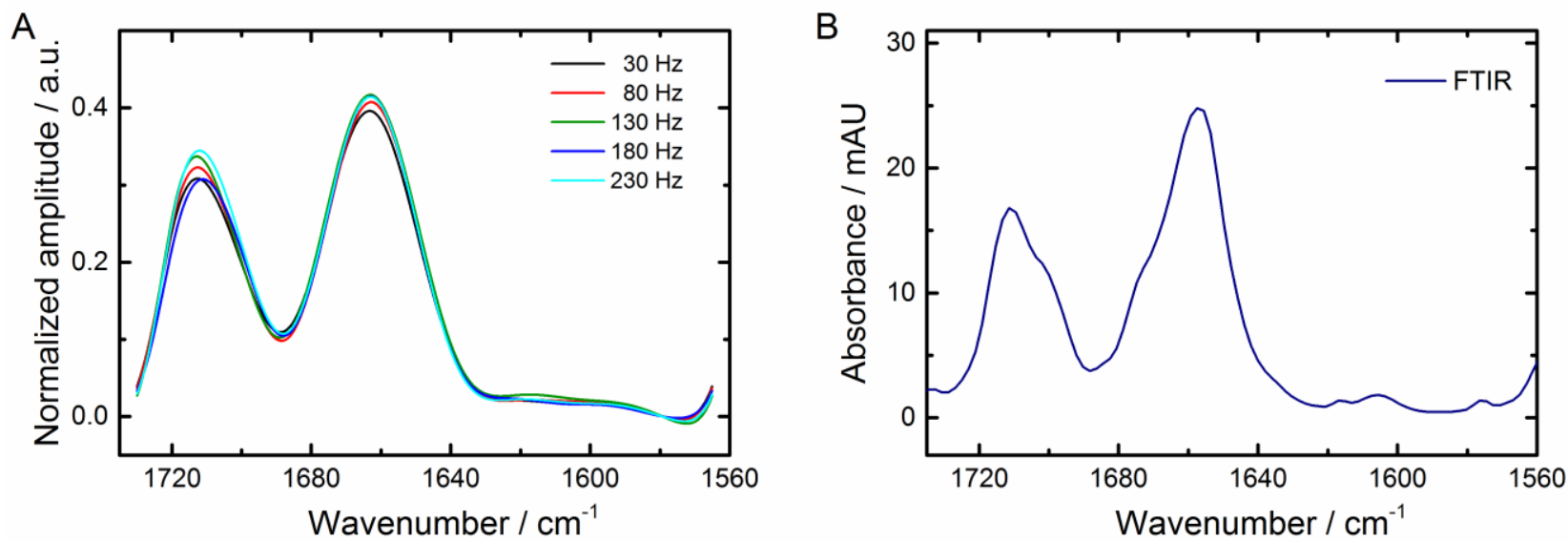

Figure 6: Normalized PTI spectra of $1.5 \mathrm{~g} \mathrm{~L}^{-1}$ caffeine in ethanol measured at different modulation frequencies. (B) Reference spectrum of caffeine in ethanol recorded by FTIR spectroscopy. 

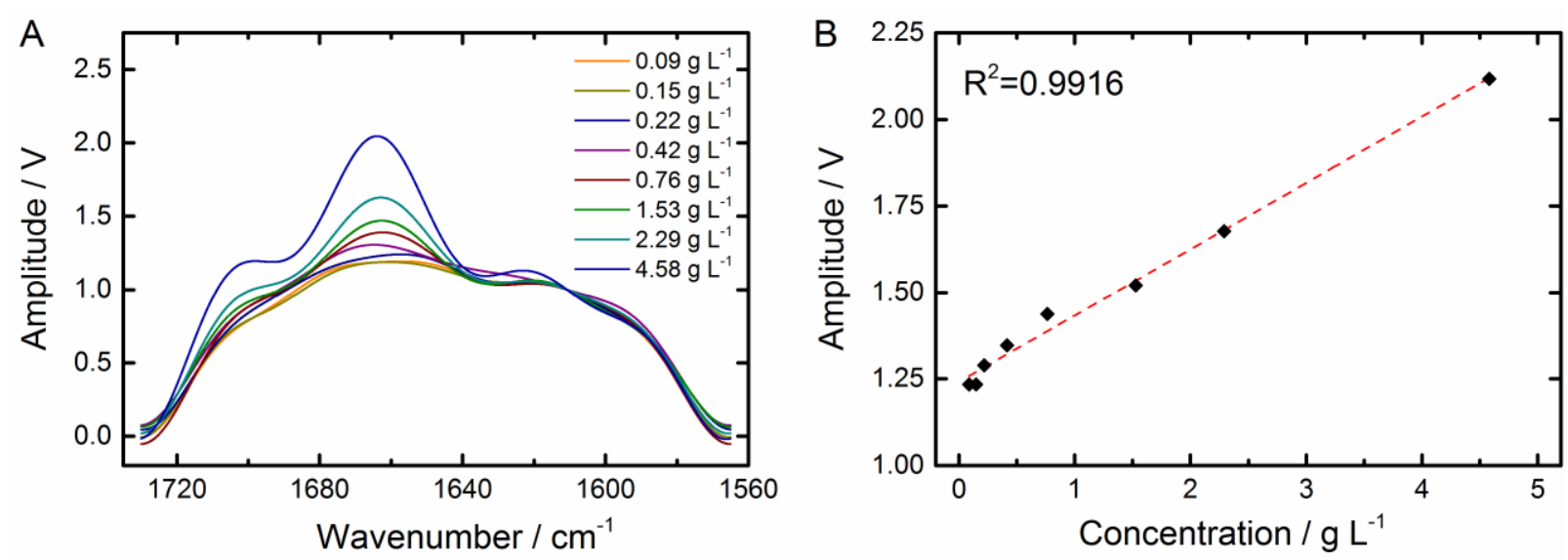

Figure 7: (A) PTI amplitude spectra of different caffeine concentrations in ethanol measured at a modulation frequency of $230 \mathrm{~Hz}$. (B) Calibration curve for caffeine in ethanol. Amplitudes were read out at $1664 \mathrm{~cm}^{-1}$.

\subsection{PT-MZI spectra of casein in aqueous solution}

PT-MZI amplitude spectra were recorded of water and aqueous casein solutions with protein concentrations between 2.5 and $20 \mathrm{~g} \mathrm{~L}^{-1}$ at a modulation frequency of $130 \mathrm{~Hz}$ (Figure 8A). The spectra show similar shapes for pure water and protein solutions, not revealing specific absorption features of the protein amide I absorption band. A schematic of the protein primary structure is shown in Figure 3B. The calibration curve shows a linear progression with a negative slope (Figure 8B). This behavior indicates that the recorded PTI amplitude spectra do not represent absorption by the protein itself, but rather solely constitute the absorption of water. Even though the absorption coefficient of water in this spectral region is significantly lower than the one of the protein amide I band $\left(\sim 100 \mathrm{~L} \mathrm{~mol}^{-1} \mathrm{~cm}^{-1}\right),{ }^{23}$ due to the much higher molar concentration of water (55.56 vs. $\left.0.025\left(20 \mathrm{~g} \mathrm{~L}^{-1}\right) \mathrm{mol} \mathrm{L}^{-1}\right)$, it dominates the PTI amplitude spectrum. Thus, the linearly decreasing PTI amplitude with increasing protein concentration originates in the displacement of water and consequential reduction of the water concentration in the presence of proteins compared to pure water.
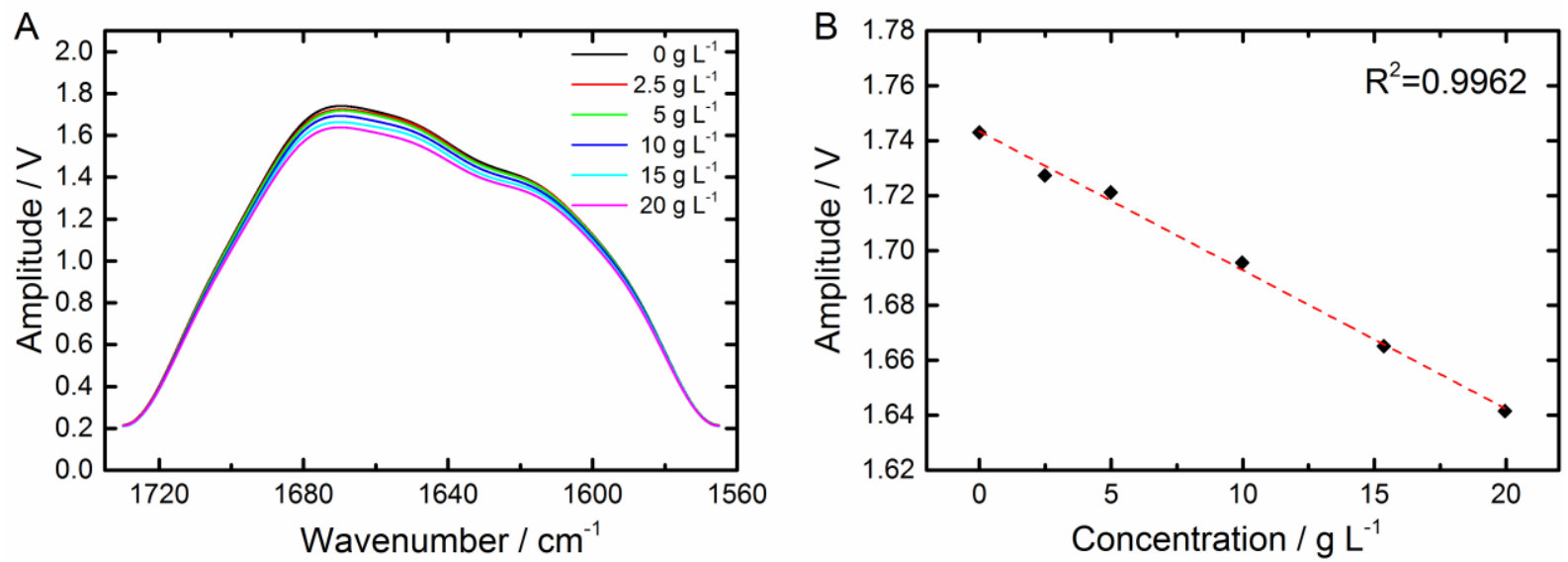

Figure 8: (A) PTI amplitude spectra of pure water and aqueous casein solutions at concentrations between 2.5 and $20 \mathrm{~g} \mathrm{~L}^{-1}$ acquired at a modulation frequency of $130 \mathrm{~Hz}$. (B) Calibration curve for casein in water. Amplitudes were read out at $1670 \mathrm{~cm}^{-1}$. 


\section{CONCLUSIONS AND OUTLOOK}

This work presents a EC-QCL-based photothermal Mach-Zehnder interferometer for phase shift interferometry of liquid samples. It has been demonstrated that this measurement principle is very well applicable for analytes of different kind. For caffeine in ethanol, PT-MZI spectra were obtained, whose bands are well comparable to conventional FTIR spectra. Furthermore, a linear relationship was shown between the PTI amplitude and the caffeine concentration at a range from 0.1 to $4.5 \mathrm{~g} \mathrm{~L}^{-1}$. An investigation of the dependency of the PTI amplitude on the modulation frequency revealed extensive influences of physical properties of the medium as reflected by theoretical considerations. Finally, in the current early state of the presented setup, PT-MZI spectra of proteins in aqueous solution do not reveal spectral features of the protein amide I band but can be employed for quantitative studies of proteins.

\section{ACKNOWLEDGEMENTS}

This work has received funding from the European Union's Horizon 2020 research and innovation programme under the grant agreement no.: 731778. M.M. gratefully acknowledges the financial support provided by CONICET and the Austrian Agency for International Cooperation in Education and Research (OeAD).

\section{REFERENCES}

[1] Ropcke, J., Davies, P. B., Lang, N., Rousseau, A. and Welzel, S., "Applications of quantum cascade lasers in plasma diagnostics: a review," J. Phys. D: Appl. Phys., 45(42), 423001 (2012).

[2] Schwaighofer, A., Brandstetter, M. and Lendl, B., "Quantum cascade lasers (QCLs) in biomedical spectroscopy," Chem. Soc. Rev., 46, 5903-5924 (2017).

[3] Li, J. S., Chen, W. and Fischer, H., "Quantum Cascade Laser Spectrometry Techniques: A New Trend in Atmospheric Chemistry," Appl. Spectrosc. Rev., 48(7), 523-559 (2013).

[4] Brandstetter, M., Genner, A., Anic, K. and Lendl, B., "Tunable external cavity quantum cascade laser for the simultaneous determination of glucose and lactate in aqueous phase," Analyst, 135(12), 3260-3265 (2010).

[5] Alcaráz, M. R., Schwaighofer, A., Kristament, C., Ramer, G., Brandstetter, M., Goicoechea, H. and Lendl, B., "External cavity-quantum cascade laser spectroscopy for mid-IR transmission measurements of proteins in aqueous solution," Anal. Chem., 87(13), 6980-6987 (2015).

[6] Schwaighofer, A., Alcaraz, M. R., Araman, C., Goicoechea, H. and Lendl, B., "External cavity-quantum cascade laser infrared spectroscopy for secondary structure analysis of proteins at low concentrations," Sci. Rep., 6, 33556 (2016).

[7] Skvortsov, L. A., "Laser photothermal spectroscopy of light-induced absorption," Quantum Electron., 43(1), 113 (2013).

[8] Pfeifer, M., Ruf, A. and Fischer, P., "Indirect absorption spectroscopy using quantum cascade lasers: mid-infrared refractometry and photothermal spectroscopy," Opt. Express, 21(22), 25643-25654 (2013).

[9] Owens, M. A., Davis, C. C. and Dickerson, R. R., "A Photothermal Interferometer for Gas-Phase Ammonia Detection," Anal. Chem., 71(7), 1391-1399 (1999).

[10] Proskurnin, M. A. and Kononets, M. Y., "Modern analytical thermooptical spectroscopy," Russ. Chem. Rev., 73(12), 1235-1268 (2004).

[11] Kim, S., Lee, D., Liu, X. C., Van Neste, C., Jeon, S. and Thundat, T., "Molecular recognition using receptor-free nanomechanical infrared spectroscopy based on a quantum cascade laser," Sci. Rep., 3, 1111 (2013).

[12] Skvortsov, L. A. and Maksimov, E. M., "Application of laser photothermal spectroscopy for standoff detection of trace explosive residues on surfaces," Quantum Electron., 40(7), 565-578 (2010).

[13] Farahi, R. H., Passian, A., Tetard, L. and Thundat, T., "Pump-probe photothermal spectroscopy using quantum cascade lasers," J. Phys. D: Appl. Phys., 45(12), 125101 (2012).

[14] Monson, B., Vyas, R. and Gupta, R., "Pulsed and cw photothermal phase shift spectroscopy in a fluid medium: theory," Appl. Opt., 28(13), 2554-2561 (1989).

[15] Rose, A., Vyas, R. and Gupta, R., "Pulsed Photothermal Deflection Spectroscopy in a Flowing Medium - a Quantitative Investigation," Appl. Opt., 25(24), 4626-4643 (1986). 
[16] Mazzoni, D. L. and Davis, C. C., "Trace Detection of Hydrazines by Optical Homodyne Interferometry," Appl. Opt., 30(7), 756-764 (1991).

[17] Faubel, W., Seidel, B. S. and Ache, H. J., "Trace analysis of water pollutants by photothermal phase shift spectroscopy with an integrated optical microinterferometer," Opt. Eng., 35(12), 3555-3561 (1996).

[18] Reider, G. A., [Photonik: Eine Einführung in die Grundlagen], Springer Vienna, Vienna, (2005).

[19] Taeye, J. D. and Huyskens, T. Z., "Infrared Spectrum of Caffeine and its Hydrochloride Dihydrate," Spectrosc. Lett., 19(4), 299-310 (1986).

[20] Venyaminov, S. Y. and Prendergast, F. G., "Water (H2O and D2O) molar absorptivity in the 1000-4000 cm(-1) range and quantitative infrared spectroscopy of aqueous solutions," Anal. Biochem., 248(2), 234-245 (1997).

[21] Bashkatov, A. N. and Genina, E. A., "Water refractive index in dependence on temperature and wavelength: a simple approximation," Proc. Soc. Photo-Opt. Instrum. Eng., 5068, 393-395 (2002).

[22] Rioboo, R. J. J., Philipp, M., Ramos, M. A. and Kruger, J. K., "Concentration and temperature dependence of the refractive index of ethanol-water mixtures: Influence of intermolecular interactions," Eur. Phys. J. E, 30(1), 1926 (2009).

[23] Tamm, L. K. and Tatulian, S. A., "Infrared spectroscopy of proteins and peptides in lipid bilayers," Q. Rev. Biophys., 30(4), 365-429 (1997). 